DOI: $10.17516 / 1997-1397-2020-13-5-568-573$

УДК 517.9

\title{
On a Problem that Does not Have Basis Property of Root Vectors, Associated with a Perturbed Regular Operator of Multiple Differentiation
}

\author{
Nurlan S. Imanbaev* \\ South Kazakhstan State Pedagogical University \\ Shymkent, Kazakhstan \\ Institute of Mathematics and Mathematical Modeling \\ Almaty, Kazakhstan
}

Received 18.05.2020, received in revised form 30.06.2020, accepted 04.07.2020

\begin{abstract}
A spectral problem for a multiple differentiation operator with integral perturbation of boundary value conditions which are regular but not strongly regular is considered in the paper. The feature of the problem is the absence of the basis property of the system of root vectors. A characteristic determinant of the spectral problem is constructed. It is shown that absence of the basis property of the system of root functions of the problem is unstable with respect to the integral perturbation of the boundary value condition.
\end{abstract}

Keywords: multiple differentiation operator, integral perturbation of boundary value conditions, basis property, root vectors, system of eigenfunctions and associated functions, eigenvalue, characteristic determinant.

Citation: N.S. Imanbaev, On a Problem that Does not Have Basis Property of Root Vectors, Associated with a Perturbed Regular Operator of Multiple Differentiation, J. Sib. Fed. Univ. Math. Phys., 2020, 13(5), 568-573. DOI: 10.17516/1997-1397-2020-13-5-568-573.

\section{Introduction and formulation of the problem}

It is well known that a system of eigenfunctions of an operator defined by a formally selfadjoint differential expression with arbitrary self-adjoint boundary value conditions, that provide a discrete spectrum, forms an orthonormal basis in the space $L_{2}$. The problem of preservation of basis properties with respect to some weak (in a certain sense) perturbation of the initial operator was investigated in many papers. For example, the problem was studied in the case of a self-adjoint initial operator [1-3], and in the case of a non self-adjoint initial operator [4-6]. The following spectral problem is considered in this paper (it is close to the problems considered in $[1,4,7])$

$$
\begin{gathered}
l(u) \equiv-u^{\prime \prime}(x)=\lambda u(x), \quad 0<x<1 \\
U_{1}(u) \equiv u^{\prime}(0)-u^{\prime}(1)-\alpha u(1)=0, \quad \alpha>0, \\
U_{2}(u) \equiv u(0)=\int_{0}^{1} \overline{p(x)} u(x) d x, \quad p(x) \in L_{2}(0,1) .
\end{gathered}
$$

*imanbaevnur@mail.ru https://orcid.org/0000-0002-5220-9899

(c) Siberian Federal University. All rights reserved 
Where $\lambda$ is an arbitrary complex number. Stability of the basis property of root vectors of the spectral problem has was studied in the case $\alpha=0$ [8]. It is common knowledge that system of root functions of an ordinary differential operator with arbitrary strongly regular boundary value conditions forms Riesz basis in the space $L_{2}(0,1)$. In the case when boundary value conditions are regular but not strongly regular, the basis property of systems of root functions, in contrast to the completeness property, is not even determined by the specific type of boundary value conditions. This effect was first established by V. A. Il'in [5], and the corresponding example was constructed for some second-order differential operator. In this case it was shown that not only boundary value conditions but also the values of coefficients of the differential operator affect the existence of the basis property. Moreover, this property can be changed with an arbitrarily small change in values of the coefficients in the metric of those classes in which these coefficients are given. Let $L_{1}$ be an operator in $L_{2}(0,1)$. It is defined by (1) and "perturbed" boundary value conditions

$U_{1}(u) \equiv u^{\prime}(0)-u^{\prime}(1)-\alpha u(1)=0, \quad \alpha>0, \quad U_{2}(u) \equiv u(0)=\int_{0}^{1} \overline{p(x)} u(x) d x, \quad p(x) \in L_{2}(0,1)$.

Unperturbed operator (the case $p(x)=0$ ) is denoted by $L_{0}$. Various variants of the integral perturbation of boundary value conditions were considered [9-13]. It was assumed that unperturbed operator $L_{0}$ has a system of eigenfunctions and associated functions (E\&AF) that forms the Riesz basis in $L_{2}(0,1)$. Characteristic determinant of the spectral problem for operator $L_{1}$ was also constructed. Then inferences were drawn about stability or instability of the Riesz basis property of the E\&AF problem with an integral perturbation of the boundary value condition.

The distinctive feature of this paper is that system of eigenfunctions of unperturbed problem (1)- (3) is complete but it does not form a basis in $L_{2}(0,1)$ [14]. Therefore, the method that we use in the previous papers cannot be applied in this case.

\section{Characteristic determinant of the spectral problem}

Boundary value conditions in problem (1)-(3) are regular but not strongly regular [15]. System of root functions of operator $l$ is a complete system but does not even form a usual basis in $L_{2}(0,1)$ [14]. However, as it was shown in [16], a basis can be constructed with the use of these eigenfunctions. This makes it possible to apply the method of separation of variables to solve the initial-boundary value problem with boundary value condition (2). The problem that does not have the basis property of root vectors in $L_{2}(0,1)$ defined by expression (1) with integral perturbation of boundary value condition (2) is studied [17]. The general solution of equation (1) has the form

$$
u(x, \lambda)=C_{1} \cos \sqrt{\lambda} x+C_{2} \sin \sqrt{\lambda} x .
$$

Using boundary value conditions (2)-(3), we obtain the following linear system with respect to coefficients $C_{k}$ :

$$
\left\{\begin{array}{l}
C_{1}(\sqrt{\lambda} \sin \sqrt{\lambda}-\alpha \cos \sqrt{\lambda})+(\sqrt{\lambda}(1-\cos \sqrt{\lambda})-\alpha \sin \sqrt{\lambda})=0 \\
C_{1}\left(1-\int_{0}^{1} \overline{p(x)} \cos \sqrt{\lambda} x d x\right)+C_{2} \int_{0}^{1} \overline{p(x)} \sin \sqrt{\lambda} x d x=0
\end{array}\right.
$$


Therefore, characteristic determinant of problem (1)-(3) has the form

$$
\begin{aligned}
& \Delta_{1}(\lambda)=(\sqrt{\lambda} \sin \sqrt{\lambda}-\alpha \cos \sqrt{\lambda}) \cdot \int_{0}^{1} \overline{p(x)} \sin \sqrt{\lambda} x d x- \\
& \quad-(\sqrt{\lambda}(1-\cos \sqrt{\lambda})-\alpha \sin \sqrt{\lambda})\left(1-\int_{0}^{1} \overline{p(x)} \cos \sqrt{\lambda} x d x\right) .
\end{aligned}
$$

If $p(x)=0$ then the characteristic determinant of unperturbed problem (1)-(3) is obtained. It is denoted by

$$
\Delta_{0}(\lambda)=\sqrt{\lambda}(1-\cos \sqrt{\lambda})-\alpha \sin \sqrt{\lambda}
$$

Solving the equation $\Delta_{0}(\lambda)=0$, we have two series of eigenvalues $\lambda_{k}^{(1)}=(2 \pi k)^{2}, k=1,2, \ldots$, $\lambda_{k}^{(2)}=\left(2 \beta_{k}\right)^{2}, k=1,2, \ldots$, of unperturbed problem (1)-(3). Here $\beta_{k}$ are roots of the equation

$$
\operatorname{tg} \beta=\frac{\alpha}{2 \beta}, \quad \beta>0 .
$$

They are positive and satisfy the following inequalities:

$\pi k<\beta_{k}<\pi k+\frac{\pi}{2}, k=0,1,2, \ldots$

For the difference $\delta_{k}=\beta_{k}-\pi k$ at large enough $k$ the following two-sided estimates are true

$$
\frac{\alpha}{2 \pi k}\left(1-\frac{1}{2 \pi k}\right)<\delta_{k}<\frac{\alpha}{2 \pi k}\left(1+\frac{1}{2 \pi k}\right) .
$$

When $p(x)=0$ eigenfunctions of the unperturbed problem have the following form

$$
u_{k}^{(1)}(x)=\sin (2 \pi k x), \quad k=1,2, \ldots, \quad u_{k}^{(2)}(x)=\sin \left(2 \beta_{k} x\right), \quad k=1,2, \ldots
$$

When $p(x)=0$ let us consider the problem adjoint to unperturbed problem (1)-(3)

$$
l *(\nu)=\bar{\lambda} \nu, v^{\prime}(1)+\alpha v(0)=0 ; \quad v(0)-v(1)=0
$$

It has eigenfunctions

$$
\begin{array}{ll}
v_{k}^{(1)}(x)=C_{k}^{(1)}\left(\cos (2 \pi k x)-\frac{\alpha}{2 \pi k} \sin (2 \pi k x)\right), & k=1,2, \ldots, \\
v_{k}^{(2)}(x)=C_{k}^{(2)}\left(\cos \left(2 \beta_{k} x\right)+\frac{\alpha}{2 \beta_{k}} \sin \left(2 \beta_{k} x\right)\right), \quad k=1,2, \ldots,
\end{array}
$$

where $C_{k}^{(1)}, C_{k}^{(2)}$ are selected from the ratio of biorthogonality $\left(u_{k}^{(1)}, v_{k}^{(1)}\right)=1,\left(u_{k}^{(2)}, v_{k}^{(2)}\right)=$ 1. Hence,

$$
C_{k}^{(1)}=-\frac{4 \pi}{\alpha}, \quad C_{k}^{(2)}=\frac{4 \pi}{\alpha}+O\left(\frac{1}{k}\right) .
$$

An auxiliary system was constructed that forms the basis in $L_{2}(0,1)$ [16]

$$
\begin{gathered}
u_{0}(x)=u_{0}^{(2)}(x) \cdot\left(2 \beta_{0}\right)^{-1}, \quad u_{2 k}(x)=u_{k}^{(1)}(x), \\
u_{2 k-1}(x)=\left(u_{k}^{(2)}(x)-u_{k}^{(1)}(x)\right) \cdot\left(2 \delta_{k}\right)^{-1}, \quad k=1,2, \ldots .
\end{gathered}
$$

The system that is biorthogonal to auxiliary system is

$$
v_{0}(x)=2 \beta_{0} v_{0}^{(2)}(x), \quad v_{2 k}(x)=v_{k}^{(2)}(x)+v_{k}^{(1)}(x), \quad v_{2 k-1}(x)=2 \delta_{k} v_{k}^{(2)}(x), \quad k=1,2, \ldots
$$


It is constructed from the eigenfunctions of problem (8). The function $p(x)$ can be represented in terms of Fourier series based on the auxiliary system $\left\{v_{k}(x)\right\}$ :

$$
p(x)=a_{0} v_{0}(x)+\sum_{k=1}^{\infty}\left(a_{k} v_{2 k}(x)+b_{k} v_{2 k-1}(x)\right) .
$$

We integrate expression (5) and obtain

$$
\begin{aligned}
\int_{0}^{1} \overline{p(x)} \sin \sqrt{\lambda} x d x=\Delta_{0}(\lambda) \cdot\left\{\bar{a}_{0} \cdot C_{0}^{(2)} \frac{2 \beta_{0}}{\lambda-\left(2 \beta_{0}\right)^{2}}+\right. \\
\left.+\sum_{k=1}^{\infty} \bar{a}_{k} \cdot C_{k}^{(1)} \frac{1}{\lambda-(2 k \pi)^{2}}+\sum_{k=1}^{\infty} \frac{C_{k}^{(2)}}{\lambda-\left(2 \beta_{k}\right)^{2}}\left(\bar{a}_{k}+\bar{b}_{k}\left(2 \delta_{k}\right)\right)\right\},
\end{aligned}
$$

where

$$
\begin{gathered}
\Delta_{0}(\lambda)=\sqrt{\lambda}(1-\cos \sqrt{\lambda})-\alpha \sin \sqrt{\lambda} \\
\int_{0}^{1} \overline{p(x)} \cos \sqrt{\lambda} x d x=\bar{a}_{0} \frac{\left(2 \beta_{0}\right) \cdot C_{0}^{(2)}}{\lambda-\left(2 \beta_{0}\right)^{2}}(\sqrt{\lambda} \sin \sqrt{\lambda}-\alpha \cos \sqrt{\lambda})+ \\
+\sum_{k=1}^{\infty} \bar{a}_{k} \frac{C_{k}^{(1)}}{\lambda-(2 k \pi)^{2}}(\alpha(1-\cos \sqrt{\lambda})+\sqrt{\lambda} \sin \sqrt{\lambda})+ \\
+\sum_{k=1}^{\infty}\left(\bar{a}_{k}+\bar{b}_{k}\left(2 \delta_{k}\right)\right) \frac{C_{k}^{(2)}}{\lambda-\left(2 \beta_{k}\right)^{2}}(\sqrt{\lambda} \sin \sqrt{\lambda}-\alpha \cos \sqrt{\lambda}-\alpha) .
\end{gathered}
$$

Using obtained results, determinant (5) is reduced to the form

$$
\begin{aligned}
\Delta_{1}(\lambda)=\Delta_{0}(\lambda) & {\left[-1+\alpha \sum_{k=1}^{\infty}\left(a_{k}\left(C_{k}^{(1)} \frac{1}{\lambda-(2 k \pi)^{2}}-C_{k}^{(2)} \frac{1}{\lambda-\left(2 \beta_{k}\right)^{2}}\right)-\right.\right.} \\
- & \left.b_{k} \frac{2 \delta_{k} C_{k}^{(2)}}{\lambda-\left(2 \beta_{k}\right)^{2}}\right)+2(\sqrt{\lambda} \sin \sqrt{\lambda}-\alpha \cos \sqrt{\lambda}) \cdot\left(a_{0} \frac{\left(2 \beta_{0}\right) \cdot C_{0}^{(2)}}{\lambda-\left(2 \beta_{0}\right)^{2}}+\right. \\
& \left.\left.+\sum_{k=1}^{\infty}\left(a_{k}\left(C_{k}^{(1)} \frac{1}{\lambda-(2 k \pi)^{2}}+C_{k}^{(2)} \frac{1}{\lambda-\left(2 \beta_{k}\right)^{2}}\right)+b_{k} \frac{\left(2 \delta_{k}\right) C_{k}^{(2)}}{\lambda-\left(2 \beta_{k}\right)^{2}}\right)\right)\right],
\end{aligned}
$$

where $\Delta_{0}(\lambda)=\sqrt{\lambda}(1-\cos \sqrt{\lambda})-\alpha \sin \sqrt{\lambda}$

Expression in square brackets is denoted by $A(\lambda)$. The obtained above result is formulated as the following theorem.

Theorem 2.1 Characteristic determinant of spectral problem (1)-(3) with perturbed boundary value conditions can be represented in form $(11)$, where $\Delta_{0}(\lambda)$ is the characteristic determinant of the unperturbed problem, and $a_{k}, b_{k}$ are the Fourier coefficients of expansion (10) of the function $p(x)$ in the biorthogonal system of eigenfunctions of adjoint problem (8).

Function $A(\lambda)$ with $\lambda=\left(2 \beta_{k}\right)^{2}$ and $\lambda=(2 \pi k)^{2}$ has first order poles. Therefore, $\Delta_{1}(\lambda)=$ $=\Delta_{0}(\lambda) \cdot A(\lambda)$ is an entire analytic function of the variable $\lambda$. Characteristic determinant (11) 
has the simple form when $p(x)$ is represented as a finite sum in (10). When there exists a number $N$ such that $a_{k}=b_{k}=0$ for all $k>N$ then function $A(\lambda)$ has the form

$$
\begin{aligned}
& A(\lambda)=-1+ \alpha \sum_{k=1}^{N}\left[a_{k}\left(C_{k}^{(1)} \frac{1}{\lambda-(2 k \pi)^{2}}-C_{k}^{(2)} \frac{1}{\lambda-\left(2 \beta_{k}\right)^{2}}\right)-\right. \\
&\left.-b_{k} \frac{2 \delta_{k} C_{k}^{(2)}}{\lambda-\left(2 \beta_{k}\right)^{2}}\right]+2(\sqrt{\lambda} \sin \sqrt{\lambda}-\alpha \cos \sqrt{\lambda}) \cdot\left(a_{0} \frac{\left(2 \beta_{0}\right) \cdot C_{0}^{(2)}}{\lambda-\left(2 \beta_{0}\right)^{2}}+\right. \\
& \quad+\sum_{k=1}^{N}\left[a_{k}\left(C_{k}^{(1)} \frac{1}{\lambda-(2 k \pi)^{2}}+C_{k}^{(2)} \frac{1}{\lambda-\left(2 \beta_{k}\right)^{2}}\right)+b_{k} \frac{\left(2 \delta_{k}\right) C_{k}^{(2)}}{\lambda-\left(2 \beta_{k}\right)^{2}}\right] .
\end{aligned}
$$

It follows from (12) that $\Delta_{1}\left(\lambda_{k}^{1}\right)=\Delta_{1}\left(\lambda_{k}^{2}\right)=0$, for all $k>N$. Consequently, all eigenvalues $\lambda_{k}^{1}, \lambda_{k}^{2}, \quad k>N$ of unperturbed problem $(1)-(3)(p(x)=0)$ are eigenvalues of perturbed problem (1)-(3). Multiplicity of eigenvalues $\lambda_{k}^{1}, \lambda_{k}^{2}, k>N$ is preserved. It follows from the condition of biorthogonality of the system of eigenfunctions $\left\{u_{k}^{(1)}(x), u_{k}^{(2)}(x)\right\}$ and $\left\{v_{k}^{(1)}(x), v_{k}^{(2)}(x)\right\}$ that $\int_{0}^{1} \overline{p(x)} u_{k}^{(1)}(x) d x=0, \int_{0}^{1} \overline{p(x)} u_{k}^{(2)}(x) d x=0, k>N$. Thus, eigenfunc-

tions $\left\{u_{k}^{(1)}(x), u_{k}^{(2)}(x)\right\}, k>N$ of unperturbed problem $(p(x)=0)(1)-(3)$ satisfy the boundary value conditions of perturbed problem (1)-(3). Therefore, the system of eigenfunctions of perturbed problem (1)-(3) and the system of eigenfunctions of the unperturbed problem $(p(x)=0)$ coincide which do not form a basis with the exception of a finite number of the first terms. Then the system of eigenfunctions of perturbed problem $(1)-(3)$ is also not a basis in $L_{2}(0,1)$ in this particular case.

\section{References}

[1] A.S.Makin, Differential Equations, 42(2006), no. 4, 599-602. DOI: $10.1134 / \mathrm{S} 0012266106040185$

[2] A.S.Markus, On decomposition of a weakly perturbedself-adjoint operator by root vectors, Reports of the AS USSR, 142(1962), no. 3, 538-541 (in Russian).

[3] N.B.Kerimov, Kh.R.Mamedov, On the Riesz basis property of root functions of some regular boundary value problems, Math. Notes., 64(1998), 483-487.

[4] A.A.Shkalikov, On basis property of eigenfunctions of ordinary differential operators with integral boundary value conditions, Vestnik $M G U$, (1982), no. 6, 12-21 (in Russian).

[5] V.A.Ilyin, L.V.Kritskov, Journal of Mathematical Sciences, 116(2003), 3489-3550. DOI: $10.1023 / \mathrm{A}: 1024180807502$

[6] N.S.Imanbaev, M.A.Sadybekov, Basis properties of root functions of loaded differential operators of the second order, Reports of the NAS RK, (2010), no. 2, 11-13 (in Russian).

[7] N.S.Imanbaev, M.A. Sadybekov, Stability of basis property of a type of problems with nonlocal perturbation of boundary conditions, AIP Conf. Proc., Vol. 1759, ICAAM, 2016, 020034-020039. 
[8] N.S.Imanbaev, International Journal of Differential Equations, 2015(2015), Article ID 641481. DOI: $10.1155 / 2015 / 641481$

[9] N.S.Imanbaev, M.A. Sadybekov, On instability of basis property of root vectors system of the double differentiation operator with an integral perturbation of periodic type conditions, AIP Publishing: Advancements in Mathematical Sciences, 1676(2015), 020083-020088.

[10] M.A.Sadybekov, N.S.Imanbaev, Differential Equations, 48(2012), no. 6, 896-900. DOI: $10.1134 /$ S0012266112060146

[11] M.A. Sadybekov, N.S.Imanbaev, On spectral properties of a periodic problem with an integral perturbation of the boundary condition, Eurasian Mathematical Journal, 4(2013), no. $3,53-62$.

[12] N.S.Imanbaev, Stability of the basis property of eigenvalue systems of Sturm-Liouville operators with integral boundary condition, ElectronicJournal of Differential Equations, 2016(2016), no. 87, 1-8.

[13] M.A.Sadybekov, N.S.Imanbaev, Regular differential operator with a perturbed boundary condition, Mathematical Notes, 101(2017), no. 5, 878-887.

[14] P.Lang, J.Locker, Spectral theory of two-point differential operators determined by -D2, Journal Math. Anal.Appl., 146(1990), no. 1, 148-191.

[15] M.A.Naimark, Linear Differential Operators, George G. Harrap \& Company, London, 1967.

[16] A.Yu.Mokin, Differential equations, 45(2009), no. 1, 123-137.

DOI: $10.1134 /$ S0012266109010133

[17] M.A.Sadybekov, N.S.Imanbaev, Characteristic determinant of a boundary value problem, which does not have the basis property, Eurasian Math. J., 8(2017), no. 2, 40-46.

\title{
Об одной задаче, не обладающей свойством базисности корневых векторов, связанной с возмущенным регулярным оператором кратного дифференцирования
}

\author{
Нурлан С. Иманбаев \\ Южно-Казахстанский государственный педагогический университет \\ Чимкент, Казахстан \\ Институт математики и математического моделирования \\ Алма-Ата, Казахстан
}

\begin{abstract}
Аннотация. В работе рассматривается спектральная задача для оператора кратного дифференцирования при интегральном возмущении краевых условий одного типа, являющихся регулярными, но не усиленно регулярными, где особенностью задачи считают отсутствие свойства базисности у системы корневых векторов. Построен характеристический определитель спектральной задачи. Показано, что отсутствие базисности у системы корневых функций задачи неустойчиво относительно интегрального возмущения краевого условия.

Ключевые слова: оператор кратного дифференцирования, интегральное возмущение краевых условий, базисность, корневые векторы, система собственных и присоединенных функций, собственное значение, характеристический определитель.
\end{abstract}

